\title{
LA CREENCIA Y SU CONEXIÓN CON LOS ACTOS LINGÜÍSTICOS
}

SALMA SAAB

INSTITUTO de INVESTIGACIONES Filosóficas

Universidad Nacional Autónoma de MÉxico

A cincuenta años de la muerte de Freud, ha dejado de resultarnos novedoso pensar que lo mental no sólo está configurado por lo consciente y por lo que existe en el lapso en el que somos conscientes. Este cambio en la concepción de lo mental ha dado lugar a que se quieran explicar los fenómenos mentales recurriendo al modelo de las disposiciones para dar cuenta de algunos estados mentales, entre ellos, las creencias, las intenciones y las expectativas. Eligen este modelo disposicional los que propugnan por la existencia de los estados inconscientes, así como los defensores del conductismo filosófico - por ejemplo, el que defiende Ryle - para dar cuenta de la conducta observable. El modelo disposicional es uno de los modelos que se utilizan en las ciencias y tiene la característica de postular hipótesis, en términos de propiedades o de propensiones de los objetos, con el propósito de explicar lo que se observa. Si bien todavía existen muchas cuestiones que están por resolverse en torno a este modelo, no han constituido un obstáculo para que muchos filósofos recurran a él con el objeto de explicar los fenómenos mentales. Estos autores - por lo general- han pasado por alto una de las recomendaciones que con más insistencia hizo Wittgenstein a los filósofos, a saber, ser cautelosos con el uso de las analogías y la aplicación de modelos en contextos distintos a los que les dieron origen. El peligro mayor radica, según Wittgenstein, en generar discusiones fútiles que no hacen más que producir confusión en el tratamiento de problemas ya de por sí difíciles, y que obligan al filósofo a darse a la tarea de disolver el caos que otros filósofos han producido. Muy en particular señala la tendencia de los filósofos a querer dar explicaciones en lugar de quedarse en el nivel de las descripciones. El caso de la creencia, que aquí nos ocupa, sería para Wittgenstein un ejemplo de esos tantos casos.

La creencia es uno de los estados mentales en los que más claramente se aprecia el vínculo entre el pensamiento y el habla, y en forma más general, entre el pensamiento y las acciones. Wittgenstein admite que la creencia es una disposición, pero en la medida en que es fiel a su propia recomendación de no 
dar explicaciones, no se pronuncia respecto a una serie de cuestiones que en la actualidad ocupan un lugar prominente, como por ejemplo la relación entre lo mental y su base neurofisiológica y el tipo de leyes -en caso de que existanque las conectan. Dice poco respecto a estas cuestiones ya que considera que no son de su competencia en cuanto filósofo. Sus breves comentarios acerca de la conexión entre el nivel neurofisiológico y el nivel mental son negativos: considera que no se trata ni de una relación de identidad, ni tampoco de un paralelismo psico-físico. En relación con esta última, Wittgenstein sostiene que ciertos fenómenos psicológicos no pueden estudiarse fisiológicamente, por el simple hecho de que no habría nada fisiológico que les correspondiera. Escribe:

No supposition seems to me more natural than that there is no process in the brain correlated with associating or with thinking; so that it would be impossible to read off thought processes from brain-processes. I mean this: if I talk or write there is, I assume, a system of impulses going out from the brain and correlated with my spoken or written thoughts. But why should the system continue further in the direction of the centre? Why should this order not proceed, so to speak, out of chaos? The case would be like the following -certain kinds of plants multiply by seed, so that a seed always produces a plant of a same kind as that from which it was produced - but nothing in the seed corresponds to the plant which comes from it; so that it is impossible to infer the properties or structure of the plant from those of the seed that it comes out of - this can only be done from the history of the seed. So an organism might come into being even out of semetbing quite amorphous, as it were causelessly; and there is no reason why this should not really bold for our thoughts, and bence for our talking and writing. ${ }^{1}$

Así pues, el interés de Wittgenstein se dirigirá a la relación específica entre lo mental y sus manifestaciones externas, otro de los aspectos que más se han enfatizado en la discusión contemporánea, sobre todo por quienes defienden la teoría disposicional. Sin embargo, quienes proponen el modelo disposicional no se ponen de acuerdo sobre el tipo de vínculo que se establece entre lo mental y su manifestación externa. Para algunos, se trata de un vínculo interno o lógico, mientras que, para otros, es externo y causal y para los menos, se trata de uno o de otro dependiendo de cómo se describan los sucesos. Los proponentes más destacados de esta última son D. Davidson y, en sus escritos más recientes, D. Armstrong. Wittgenstein se incluiría entre los segundos - los que aceptan que los estados mentales se conectan con la acción, en particular con los actos lingüísticos, de manera interna o lógica. En este trabajo intentaré aclarar en qué consiste la tesis expresiva del lenguaje, y cómo ésta podría interpretarse como su manera de defender el modelo disposicional. Aludiré a lo que se conoce como la Paradoja de Moore, en torno a la cual

1 Remarks on the Philosophy of Pyychology, vol. I, Oxford, Blackwell, 1980, §903. 
Wittgenstein discute uno de sus comentarios más importantes acerca de la creencia, a saber, la equivalencia de "Yo creo que $p$ " y " $p$ " al ser expresadas por un sujeto particular.

A pesar de que Wittgenstein acepta que la creencia es una disposición del sujeto, ya que tiene creencias aun cuando no esté ocupándose en ese momento de ellas, sus comentarios más relevantes tienen que ver con las situaciones en las que el sujeto es consciente de sus creencias. Le interesa destacar ciertas similitudes que existen entre las creencias como ocurrencias presentes y otras experiencias como las sensaciones y las emociones. También le interesan las creencias conscientes, ya que son las que se revelan en las manifestaciones verbales, como actos intencionales, ya que las creencias inconscientes, por el contrario, suelen manifestarse en la conducta no verbal.

La postura de Wittgenstein acerca de la creencia podría ubicarse dentro de las teorías disposicionales no reductivistas: ni reduce los fenómenos mentales a la conducta, ni los identifica con procesos neurofisiológicos. Wittgenstein se vale del contraste entre la perspectiva de la primera persona y la de la tercera persona para decir que en las afirmaciones en primera persona respecto de las creencias propias, más que ser descriptivas o informativas de lo que el sujeto cree, son expresivas. Wittgenstein también negará que esos actos lingüísticos sean efecto o resultado de la creencia en cuestión.

La renuencia de Wittgenstein a considerar la relación causalmente, deriva de que si así lo fuera, sería posible que la causa existiera sin el efecto y viceversa. Wittgenstein requiere un vínculo no independiente entre el estado mental y su manifestación externa, entre otras cosas, para posibilitar que los demás puedan hacer atribuciones de creencias al sujeto a partir de la observación de sus manifestaciones externas. La manifestación externa como "expresión" de lo que sucede internamente, nos permite reconocerla como su "expresión natural", y así justificar nuestra atribución. Wittgenstein no cree posible que uno capture el estado mental, y que una vez capturado, pudiera asociarle una expresión: el vínculo, más bien, tiene que ser interno. La tesis de la expresividad de la conducta verbal y no verbal respecto a los estados internos, constituye uno de los aportes más originales de Wittgenstein, a la vez que uno de los más controvertidos. Pero veamos qué entiende Wittgenstein por "expresión". Una característica de la expresión, que en el caso de las sensaciones es más patente, es que no es posible tener la sensación sin su expresión correspondiente. ${ }^{2}$ Una expresión natural del dolor, por ejemplo, es que la persona grite, se retuerza, haga ciertas gesticulaciones, etc. Estas expresiones naturales, una vez que se

2 Ibid., §308-10. 
adquieren las capacidades lingüísticas, son reemplazadas por expresiones verbales, y en cuanto sustituciones también serán expresión de la sensación. Dice Wittgenstein: "La expresión natural del dolor reemplaza al grito y no lo describe." 3 Sin embargo, Wittgenstein concede que no contamos con manifestaciones naturales para cada uno de nuestros estados mentales, ya que una misma expresión podría estar asociada a diferentes conceptos, y pone como ejemplo la expectativa. ${ }^{4}$

Wittgenstein considera que la creencia cuenta con una expresión natural, que es una expresión verbal: la expresión "yo creo que...". Sostiene que cuando una persona se autoadscribe una creencia, esta afirmación nos arroja luz sobre el estado de la persona que lo dice, y nos permite extraer conclusiones a partir de lo que dice. La expresión de nuestras creencias en la primera persona no es informativa o descriptiva. Wittgenstein entiende la relación descriptiva de manera semejante a la relación referencial entre nombre y cosa nombrada. En una situación descriptiva, el sujeto observa la situación objetiva y como resultado de la observación describe la situación. Otra característica de las descripciones es que cuentan con criterios que nos permiten que juzguemos si la descripción es verdadera o falsa.

En el contexto de la discusión de las sensaciones, Wittgenstein nos dice cómo es que las palabras se refieren a las sensaciones, que no debe confundirse con la manera como se refieren las palabras a objetos externos, a saber, como si se tratara de una relación entre nombres y cosas nombradas. Las palabras que se refieren a estados internos no describen porque esto supondría que se refieren a ciertos objetos, a saber, sensaciones o estados, y para Wittgenstein es incorrecto ya que supondría que el sujeto "observa" lo que sucede internamente y, como resultado de esa observación, le asocia la palabra. Pero para él, esa supuesta observación no tiene por qué ser reglamentaria: a veces se daría y a veces no. Me parece que aquí radica la razón central de Wittgenstein para negarles a las expresiones verbales el estatuto de descripciones: si lo fuesen nos comprometería a pensar en un referente - que en el caso de la creencia sería un pensamiento- al cual aludieran esos actos verbales. No se requiere que el pensamiento esté presente para luego extraer o derivar de él su descripción.

Algunos consideran que la tesis de Wittgenstein es la tesis fuerte de que las autoadscripciones que hacen referencia a estados mentales nunca tendrian carácter descriptivo. Consideran que si bien es un acierto de Wittgenstein hablar de usos expresivos asociados a los actos lingüísticos, es erróneo, sin embargo, no adjudicarles también un uso descriptivo. Me parece que Wittgen-

3 Investigaciones filos bficas, Instituto de Investigaciones Filosóficas, UNAM - Editorial Crítica, México-Barcelona, 1988, $\S 244$, y Remarks..., $\$ 313$.

4 Cf. Investigaciones filosóficas, $\S 577$. 
stein no niega que haya usos descriptivos, aunque tal vez no conceda que estos usos sean muy frecuentes. Pero aquí debemos tener cuidado de separar la tesis expresiva cuando se refiere a las sensaciones y a las emociones, y cuando se reficre a los casos como la creencia. En los primeros, Wittgenstein es explícito en que el uso expresivo - tanto verbal como no-verbal- es el predominante, pero no es claro que lo sostenga con esa generalidad para el caso de las creencias. No obstante, me parece correcto el comentario que hace P. Strawson en el sentido de que Wittgenstein exagera el énfasis en los usos expresivos, ya que nos hacen suponer que los actos verbales se nos imponen, cuando muy frecuentemente son actos intencionales que tienen el propósito de comunicar y de informar a los otros lo que creemos. 5

Wittgenstein no explora el concepto de la creencia con la profusión y el interés con los que, al hablar del lenguaje privado, explora otros conceptos mentales. Encuentra más semejanza entre el concepto de la creencia y los conceptos de tener la expectativa, desear y entender. ${ }^{6}$ La razón pareciera ser que una misma proposición puede ser manifestación de cualquiera de ellas, además de que para que se considere que se trata de cualquiera de esos estados, no es necesario que el sujeto en ese momento esté pensando en ello. Lo importante para Wittgenstein no es lo que pasa en ese momento, sino la circunstancia que rodea al fenómeno: lo que pasa antes y después. Sus comentarios más importantes al respecto se hallan en la parte segunda de las Investigaciones filosóficas, en los comentarios preparatorios a esta obra, recogidos en los Remarks on the Philosophy of Pyychology y en Zettel. ${ }^{7}$ En las Investigaciones filosóficas Wittgenstein escribe:

Pienso esto: creer es un estado mental. Tiene duración; y es independiente de la duración de su expresión en una oración, por ejemplo. Es por tanto una especie de disposición del que cree. Se me revela, en el orro, por su conducta; y por sus palabras. Tanto por la expresión "Yo creo que..." lo mismo que por la simple aserción. - ¿Qué pasa en mi propio caso: cómo reconozco yo mi propia disposición?- iYo tendría que hacer como el otro, ponerme atención, escuchar mis palabras, ser capaz de sacar de ellas conclusiones!

Yo tengo hacia mis propias palabras una relación completamente distinta de la de los demás.

Si yo pudiera decir "Me parece que creo", ese otro desarrollo del verbo hubicra sido posible. ${ }^{8}$

5 Cf. "Review of Philosophical Investigations", en Wittgenstein, G. Pitcher (ed.), MacMillan \& Co. Ltd., Londres, 1968, pp. 55-6.

6 Cf. Investigaciones filosoficas, $§ 573$.

7 Zettel, Instituto de Investigaciones Filosóficas, UNAM, México; 1985.

8 Ibid., X, p. 192. 
Wittgenstein señala claramente el contraste que priva entre el acceso que el sujeto tiene respecto a sus propias creencias y la manera en que acceden a ellas los demás. A la vez, cuestiona que la diferencia entre la perspectiva en la primera persona y la perspectiva en la tercera persona vaya acompañada del contraste entre el autoconocimiento y el conocimiento de los demás. Wittgenstein le arrebata al sujeto el privilegio de tener un conocimiento directo y certero que a la vez restringía el conocimiento de los demás a un conocimiento indirecto, hipotético y expuesto a la duda. Si así fuese, el sujeto sería el árbitro exclusivo de su interioridad, árbitro infalible, empero, por ser él el que lo experimenta. Los demás tendrían que especular con base en los signos que el sujeto exterioriza. Wittgenstein también negaría la tesis que algunos han sostenido según la cual el sujeto cuenta con justificación plena con respecto a lo que pasa en su interioridad; si así fuese, el sujeto no requeriría de ningún esfucrzo para reconocer sus experiencias. Wittgenstein intenta resistir estas características epistemológicas que se asocian al contraste entre la primera y la tercera persona. Por otra parte, si bien admite que la autoadscripción no se hace con base en observaciones o criterios, y él agregaría que se hacen sin justificación, no se deberá a que la relación con su interioridad no tenga fallas por ser inmediata y privada. El juego del lenguaje de la autoatribución supone, para Wittgenstein, un aprendizaje previo en que el sujeto, en situaciones semejantes a la suya, concuerda con otros al hacer atribuciones de estados mentales.

Para Wittgenstein, el que los demás estén impedidos -necesariamente impedidos - para experimentar lo que el otro experimenta, no obstaculiza que los demás puedan juzgar acerca de lo que les es oculto. Por el contrario, habría ocasiones en que su juicio sería acertado y no así el del que lo experimenta. La asimetria de perspectiva no le da al sujeto mismo garantías y a los otros desventajas. ${ }^{9}$ Wittgenstein está en situación de restarle al sujeto autoridad en el momento en que establece el vínculo entre lo interno y sus manifestaciones externas.

Pasemos ahora a la consideración de la tesis wittgensteiniana de que la expresión "Yo creo que $p$ " tiene el mismo significado que la aserción de " $p$ ", la combinación de esta tesis con la tesis expresiva del lenguaje, y la repercusión de ambas tesis en su apreciación de la Paradoja de Moore.

Para Wittgenstein hay casos - los más usuales-en los que la expresión "Yo creo que $p$ ". puede identificarse con la aserción de " $p$ ". La sustitución se hace con base en que "reaccionamos más o menos de la misma manera cuando al-

${ }^{9}$ Cf. Remarks..., $\$ 138$. 
guien dice la primera que cuando dice la segunda"10 o a que tienen el mismo uso. En la terminología de Rosenthal ${ }^{11}$, equivaldría a decir que tienen las mismas condiciones de asertabilidad. La sustitución de las dos expresiones permite a Wittgenstein quitarle a la expresión "Yo creo que $p$ " el carácter autorreferencial que le han otorgado muchos autores, destacándose entre ellos a Descartes. Ya vimos que el modo referencial - según Wittgenstein- nos lleva erróneamente a suponer que hay un sujeto, el hablante, el cual se refiere a su estado mental de creencia. En Wittgenstein el acento está en que el sujeto no se refiere a su estado mental descriptivamente, en el sentido antes especificado, aunque no niega que el estado del sujeto se expresa o se pone de manifiesto en su afirmación.

La situación del sujeto que hace la aseveración no es análoga a la de los observadores externos en tanto que para hacer la aseveración no observa su conducta ( $y$ en consecuencia no establece ninguna hipótesis), y no podría decir, dándole algún matiz de incertidumbre, "Me parece que creo". Pero de igual manera que el sujeto no observa su propia conducta, tampoco acepta Wittgenstein que el sujeto - por lo general- "vea internamente" su estado mental.

Ahora bien, dado el caso de que una persona llegara a formarse la hipótesis de que cree que $p$, sería ilegítimo, según Wittgenstein, sustituir "yo creo que $p$ " por " $p$ ": la hipotesis de que yo crea que $p$ no es equivalente a la hipótesis de que $p$ sea el caso. ${ }^{12}$ La razón por la que la sustitución es incorrecta parece ser que la suposición de que yo creyera $p$ no tiene la función de describir, como tampoco la de expresar el estado mental en el que se encuentra el sujeto, sino la de suponer hipotéticamente que él o ella tuviera la creencia en cuestión. Vemos que esta situación hipotética claramente nos remite al sujeto y no a la hipótesis de que $p$ sea el caso.

Wittgenstein aplica la distinción entre el uso redundante y el uso no redundante de "yo creo" a la llamada Paradoja de Moore (la aserción "Yo creo que $p$, pero $p$ es falsa"). Según el uso que se dé a la expresión "yo creo" se producirá o no una contradicción. Dice Wittgenstein:

La Paradoja de Moore se puede expresar así: La aserción "Yo creo que esto es asi" se usa de manera similar a la afirmación "Esto es asi"; y, no obstante, la suposición

10 Remarks on The Philosophy of Bychology, vol. I, §477.

11 Cf. "Intentionality", en Midwest Studies in Philosophy, vol. X, P. A. French, T. E. Uehling Jr. y H. K. Wertstein (eds.), University of Minnesota Press, 1986. Las condiciones de asertabilidad se distinguen de las condiciones de verdad en el sentido de que dos proposiciones pueden tener las mismas condiciones de asertabilidad y sin embargo diferentes condiciones de verdad, como sucede justamente con el caso de "Yo creo que $p$ ” y la aserción de " $p$ " o cl caso en el que yo diga "Gracias" o que diga "Yo estoy agradecido".

12 Cf. Investigaciones filosoficas, II-X, p. 190e. 
de que yo creo que esto es así no se usa de manera similar a la suposición de que esto es así. ${ }^{13}$

En la tradición cartesiana -e incluso para Moore- lo enigmático de afirmaciones como las de la paradoja radica en que a pesar de que las aserciones son independientes entre sí - ya que una se refiere a algo interno y la otra a algo externo-, sin embargo, la unión de ambas produce una afirmación que en lugar de ser coherente y normal, como cualesquiera otras afirmaciones independientes entre sí, es absurda. Wittgenstein difiere de esos autores en dos puntos: 1) que a pesar de hablar de cosas distintas, la aserción "Yo creo que $p$ "sea independiente de la aserción de " $p$ " y 2) que su aserción conjunta produzca una aserción absurda. Wittgenstein alude a la no independencia de las dos proposiciones, por ejemplo, en el $\$ 490$ de los Remarks... :

Ésta es la paradoja: la suposición se puede expresar de la siguiente manera: "Supóngase que esto sucediera dentro de mí y aquello afuera" -pero la aserción de que esto está sucediendo dentro de mí afirma: esto está sucediendo fuera de mí. En tanto suposiciones las dos proposiciones acerca de lo de adentro y lo de afuera son totalmente independientes, pero no en tanto aserciones.

La idea de la independencia de las proposiciones hace plausible la idea - compartida por dualistas e idealistas - de que habría un discurso referido a nuestra interioridad, cuya significatividad se mantendría aun cuando no hubiese mundo externo. La no independencia de las dos aserciones también le permite a Wittgenstein explicar lo que sería inexplicable para alguien que sostuviese la independencia entre las dos proposiciones, a saber, que cuando dos personas tienen creencias contrarias, el hecho de que se refieran a algo interno, no quiere decir que no entren en conflicto y que para resolverlo nos remitamos al valor de verdad de $p$.

Wittgenstein distingue los usos hipotéticos de los usos asertivos de "Yo creo que..." dándole primacía a los primeros. Como aserción, "Yo creo que $p$ " expresa o manifiesta una actitud del sujeto respecto a $p$. Wittgenstein hace alusión a otro uso de "Yo creo que... ", que no es ni asertivo (o enfático) ni hipotético, y que se usa con el propósito de restringir o menguar el compromiso del sujeto con respecto a $p$. En este uso podría entenderse como "tal vez $p$ ", que en conjunción con la negación contundente de $p$ produce una afirmación absurda.

En las Investigaciomes filosóficas, Wittgenstein nos invita a que imaginemos un juego de lenguaje en el que la expresión "Yo creo que $p$ " sólo se usara para informarnos acerca de lo que la persona cree, esto es, usándose en forma 
descriptiva. En este caso las dos proposiciones de la afirmación compleja de Moore serían aserciones que producirían una contradicción. Cito:

Supongamos que yo introdujera una expresión -por ejemplo "Yo creo" - de la siguiente manera: debe anteponerse a los informes que sirven para decir algo acerca del que lo comunica. (La expresión, pues, no tiene por qué ir acompañada de ninguna incertidumbre. Tengamos en cuenta que la incertidumbre de la aserción también se puede expresar de modo impersonal; "Puede que hoy venga".) - "Yo creo que..., y no es asi" sería una contradicción. ${ }^{14}$

Por otra parte, Wittgenstein nos conmina a pensar en un lenguaje en el que la expresión "Yo creo que $p$ " se identificara con la aserción " $p$ ", o sea que se refiriera al contenido en lugar de referirse a la persona. En este caso habría una asimetría entre la persona que lo expresa y los otros, de manera que si los demás quisieran atribuirle a alguien una creencia de manera tentativa, no dirían "Él o ella cree" sino "Él o ella tiende o se inclina a decir...". La persona misma no tendría un uso indicativo de la expresión "Yo creo que $p$ ", pero sí tendría un uso hipotético o subjuntivo: "Supóngase que yo dijera"; "faltaría una inflexión del verbo" en el sentido de que la persona no podría decir "Yo me inclino a decir", sólo podría aseverarla. La Paradoja de Moore en una situación así no sería contradictoria, ya que el primer conyunto tendría que leerse hipotéticamente y no como una aserción. Pero entonces no se referiría hipotéticamente a la situación a la que se alude en el otro conyunto: la expresión no querría decir "Supóngase que $p$ ". ${ }^{15}$

Finalmente, en el uso tentativo de "Yo creo que..." la proposición "Podría ser que estuviera lloviendo, pero no lo está" sería ilegítima - mas no contradictoria. Wittgenstein señala que debemos cuidarnos de decir que 'podría ser que estuviera lloviendo' significa: "Yo creo que estará lloviendo", en donde lo incorrecto radica en considerar "una aserción vacilante como una aserción de vacilación." 16

Entre los pocos comentadores que se detienen en los pasajes en los que Wittgenstein considera el problema de las creencias, se encuentran Linville ${ }^{17}$ y Rosenthal. ${ }^{18}$ Linville toma los comentarios de Wittgenstein en torno a la Paradoja de Moore como un ejemplo más de perplejidad filosófica ocasionada

14 Investigaciones filosoficas, II-X, p. $191 \mathrm{e}$.

15 Cf. ibid., p. $192 \mathrm{e}$.

16 Ibid.

17 Linville, Kent, "Wittgenstein on Moore's Paradox", en Wittgenstein: Sources and Perspectives, C. G. Luckhardt (ed.), Cornell University Press, Ithaca, Nueva York, 1979.

18 Rosenthal, D. M., “Intentionality” en Midwest Studies in Philosophy, X, P. A. French, T. E. Uehling Jr. y H. K. Wettshein (eds.), University of Minnesota Press, Minneapolis, 1986. También en su "Thinking that One Thinks" (manuscrito), 1988. 
por una especie de "ceguera de algún aspecto", o a no "ver como". La ceguera en el caso de la creencia radicaría en no percatarse de las diferencias de uso entre la primera y la tercera personas. Me parece interesante enfocarlo de esa manera. Más aún, me parece que las consideraciones que aquí se señalaron podrían enriquecerse con esa perspectiva. Pero señalaré algunas diferencias. Linville piensa que si se admite que un enunciado de la forma de la Paradoja de Moore es absurdo - y no lógicamente contradictorio- nos obliga a que el primer conyunto se refiera a la persona y que la contradicción se diera sólo en caso de que se refiriera al contenido. Cito:

... in terms of language games, in nomal circumstances (whercin asserting $M$ [un ejemplo del tipo de Moore] would be "absurd") one does not conjecture or bypothesize that one believes such-and-such. If one is hypothesizing when one says "T believe it is raining, but it is not raining", then this is a hypothesis about the weather, not oneself. But when inclined to claim that the sentence "I believe it is raining, but it is not raining" asserts something logically possible, one must take the former conjunct to be about the speaker. If regarded as a truth claim about the weather, clearly such a scntence is a contradiction. Since what Moore means to be talking about in connection with such sentences is not logically impossible, wbat he understands or means by $M$ must be the hypothesis that I believe..., and it isn't so. But the expression "I believe" is not used to formulate the bypothesis that I believe. However, this expression can be seen as a bypotbesis; and it is the "dawning" of that aspect, I submit, which inclines one to claim that $M$ is logically possible. ${ }^{19}$

Me parece que el punto crucial en Wittgenstein, el cual Linville no enfatiza suficientemente, es que aun cuando se hiciese un giro en el uso de, por ejemplo, "yo creo que está lloviendo" de manera que nos refiriésemos a la persona, de igual forma ese uso no sería independiente de lo que "está sucediendo afuera", por lo que no nos salvaría de la contradicción. ${ }^{20}$ No caer en contradicción supone un tour de force mental, en el cual imagináramos circunstancias en donde tuviera sentido que yo derivara que tengo una creencia a partir de lo que digo, o que tuviera un yo dividido con lo cual pudiera darle sentido a las proposiciones del tipo de Moore. En las circunstancias normales en las que usamos la primera persona del indicativo, nuestro interés se dirige a lo que se dice y no a quien lo dice. En estas circunstancias la afirmación de Moore sería absurda, debido a que el primer conyunto expresa una creencia y el segundo conyunto describe un estado de cosas. No habría contradicción porque el primer conyunto no es descriptivo. Por otra parte, nos dice Wittgenstein que para comprender el uso hipotético de "Supóngase que yo crea...", dependemos de una comprensión previa del uso asertivo de la creencia. ${ }^{21}$

19 Op.cit., p. 296.

20 Véase la cita de las Investigaciomes filosóficas (p. 19le) antes mencionada.

21 Cf. Investigaciones filosóficas, p. 192e. 
Rosenthal, por su parte, defiende en sus escritos una explicación causal de los actos lingüísticos que expresan pensamientos. Cuando alguien dice algo con sinceridad, por lo general el acto lingüístico y el pensamiento correspondiente tienen el mismo contenido proposicional y la misma fuerza. ${ }^{22} \mathrm{Sin}$ embargo, en el tema que nos ocupa, Rosenthal sostiene 1) que la manera como transmitimos nuestro pensamiento influye en que el pensamiento y el acto linguístico tengan o no la misma fuerza y el mismo contenido proposicional: En el caso de que lo transmitamos empleando la aserción simple, tendrían la misma fuerza y contenido proposicional, mientras que si digo "Yo creo que $p$ ", son distintos; $;^{23}$ 2) en el primer caso, se estaría expresando la creencia y en el segundo se haría un reporte de que se cree $p$; 3) ambas formas tienen las mismas condiciones de asertablidad, empero diferentes condiciones de verdad. Para Rosenthal, estas tesis se ilustran claramente en los ejemplos del tipo de Moore, en los cuales no habría manera de que tuvieran condiciones coherentes de asertabilidad, ya que "el pensamiento que expreso al decir que $p$ es justamente el que niego tener al agregar que no creo que $p$ ". ${ }^{24} \mathrm{Si}$ no distinguiésemos los usos expresivos de los usos descriptivos de nuestros pensamientos, continúa Rosenthal, la Paradoja de Moore sería contradictoria y no solamente absurda. Si bien es cierto que Rosenthal recurre a la noción wittgensteineana de "expresión", sin embargo, los fines para los cuales utilizan dicha noción son distintos. A Rosenthal, por ejemplo, le interesa establecer que la relación entre el pensamiento y el habla es causal, y que no existe prioridad del pensamiento frente al habla, salvo en sentido causal. Para Rosenthal los pensamientos se expresan en el habla, ya que se vinculan causalmente. Como ya vimos, desgraciadamente Wittgenstein deja de lado esta discusión.

La identificación que hace Wittgenstein entre "Yo creo que $p$ " y la aserción de " $p$ ", debido a sus tesis respecto al significado de las expresiones y su uso, no le dejaría mucho margen para establecer una distinción entre condiciones de verdad y condiciones de asertabilidad, y dado que el uso es crucial para la semántica de los conceptos, dice Rosenthal, sería difícil no asimilar los usos expresivos y los descriptivos. ${ }^{25}$ Ciertamente, Wittgenstein, en sus últimos escritos, con su noción amplia de uso, nos permite suponer que sólo hay que atender a las condiciones de asertabilidad; pero esto no implica nada en cuanto a la distinción entre descripción y expresión. Como hemos visto en las páginas anteriores, Wittgenstein no confunde esos usos, sino que hace a los últimos menos comunes de lo que se piensa. Para Rosenthal, los ejemplos del tipo Moore son muestra patente de que existe una diferencia entre condiciones de

25 Cf. "Intentionality", op. cit., p. 156. 
verdad y condiciones de asertabilidad, diferencia que a su vez se explica por la diferencia entre descripción y expresión. Según Wittgenstein, en cambio, estos ejemplos muestran cl uso peculiar, a saber, el expresivo, que tienen las aserciones efectuadas en la primera persona del indicativo. Para él, esta tesis es independiente de la distinción entre condiciones de verdad y condiciones de asertabilidad. 